\title{
Canadian pediatric emergency physician knowledge of concussion diagnosis and initial management
}

\author{
Roger Zemek, MD*; Kaylee Eady, $\mathrm{MAPs}^{\dagger}$; Katherine Moreau, $\mathrm{PhD}^{\dagger}$; Ken J. Farion, MD*; \\ Beverly Solomon, $\mathrm{BScN}^{\ddagger}$; Margaret Weiser, $\mathrm{PhD}^{\S}$; Carol Dematteo, $\mathrm{MSc}^{\mathbb{T}}$
}

\section{ABSTRACT}

Introduction: The diagnosis of concussion is a critical step in the appropriate management of patients following minor head trauma. The authors hypothesized that wide practice variation exists among pediatric emergency medicine physicians in the application of physical and cognitive rest recommendations following an acute concussion.

Methods: The authors developed a 35-item questionnaire incorporating case vignettes to examine pediatric emergency physician knowledge of concussion diagnosis, understanding of initial management using return-to-play/school/work guidelines, use of existing concussion protocols, and perceived barriers to protocol use. Using a modified Dillman technique, the authors distributed an online survey to members of Pediatric Emergency Research Canada, a national association of pediatric emergency physicians.

Results: Of 176 potential participants, 115 (65\%) responded to the questionnaire, 89\% (95\% confidence interval [Cl]: 0.81, 0.93) of whom reported having diagnosed 20 or more concussions annually. Although $90 \%(95 \% \mathrm{Cl}: 0.83,0.94)$ of respondents adequately diagnosed concussion, only $64 \%$ (95\% Cl: $0.54,0.72$ ) correctly applied graduated return-to-play guidelines. Cognitive rest recommendations were also frequently limited: 40\% (95\% $\mathrm{Cl}: 0.31,0.49)$ did not recommend school absence, $30 \%(95 \% \mathrm{Cl}$ : $0.22,0.39)$ did not recommend schoolwork reduction, and $35 \%$ (95\% Cl: 0.27, 0.45) did not recommend limiting screen time. Eighty percent $(95 \% \mathrm{Cl}: 0.72,0.87)$ of respondents reported having used guidelines frequently or always to guide clinical decisions regarding concussion.

Conclusion: Despite a proficiency in the diagnosis of concussion, pediatric emergency physicians exhibit wide variation in recommending the graduated return to play and cognitive rest following concussion.

\section{RÉSUMÉ}

Objectif: La pose du diagnostic de commotion cérébrale constitue une étape cruciale de la prise en charge appropriée des traumas crâniens légers. Les auteurs ont émis I'hypothèse selon laquelle il existe des écarts importants de pratique parmi les urgentologues pédiatres dans l'application des recommandations concernant le repos physique et cognitif à la suite d'une commotion cérébrale aiguë.

Méthode: Les auteurs ont élaboré un questionnaire en 35 points comprenant des illustrations de cas dans le but de vérifier les connaissances des urgentologues pédiatres en matière de diagnostic de la commotion cérébrale; de compréhension de la prise en charge initiale, fondée sur des lignes directrices relatives au retour au jeu, à l'école ou au travail; d'application des protocoles existants en ce qui concerne les commotions cérébrales ainsi que d'obstacles perçus relativement à l'application des protocoles. Les auteurs ont envoyé un questionnaire en ligne, selon une version modifiée de la méthode de Dillman, aux membres du Groupe de Recherche en Urgence Pédiatrique du Canada, une association nationale d'urgentologues pédiatres.

Résultats: Sur une possibilité de 176 participants, 115 (65\%) ont répondu au questionnaire, dont $89 \%$ (IC à $95 \%$ : 0,81 $0,93)$ ont déclaré diagnostiquer 20 commotions cérébrales ou plus par année. Tandis que $90 \%$ (IC à $95 \%$ : 0,83-0,94) des répondants ont bien diagnostiqué la commotion cérébrale, seulement $64 \%$ (IC à $95 \%$ : $0,54-0,72$ ) ont appliqué correctement les lignes directrices concernant le retour progressif au jeu. Quant aux recommandations relatives au repos cognitif, elles étaient également peu appliquées dans de nombreux cas: $40 \%$ des répondants (IC à $95 \%$ : 0,31-0,49) n'ont pas recommandé I'absence à l'école; $30 \%$ (IC à $95 \%$ : 0,22 - 0,39) n'ont pas recommandé une diminution des travaux scolaires, et $35 \%$ (IC à $95 \%$ : 0,27 - 0,45) n'ont pas recommandé une limitation du temps passé à l'écran. Quatrevingts pour cent (IC à $95 \%$ : 0,72 - 0,87) des répondants ont indiqué appliquer "souvent " ou " toujours " les lignes directrices pour prendre des décisions d'ordre clinique en ce qui concerne les commotions cérébrales.

Conclusion: Malgré une connaissance approfondie des urgentologues pédiatres en matière de diagnostic de la commotion cérébrale, des écarts importants se dégagent des

From the *Departments of Pediatrics and Emergency Medicine, Children's Hospital of Eastern Ontario, University of Ottawa, ON; †Clinical Research Unit, Children's Hospital of Eastern Ontario Research Institute, University of Ottawa, ON; ¥Holland Bloorview Kids Rehabilitation Hospital, Toronto, ON; §Acquired Brain Injury Program, Parkwood Hospital; Department of Psychology, Western University, London, ON; and ISchool of Rehabilitation Science, CanChild Centre for Childhood Disability Research, McMaster University, Hamilton, ON.

Correspondence to: Dr. Roger Zemek, Children's Hospital of Eastern Ontario, 401 Smyth Road, Ottawa, ON K1H 8L1; Email: rzemek@cheo.on.ca 
recommandations en ce qui concerne le retour progressif au jeu et le repos cognitif à la suite d'une commotion cérébrale.

Keywords: Concussion, Pediatric, Adolescent, Practice Variation, Guidelines

\section{INTRODUCTION}

The diagnosis of concussion is a critical step in the appropriate management of patients following minor head trauma. ${ }^{1-4}$ The International Conference on Concussion in Sport defines concussion as a "complex pathophysiologic process affecting the brain, induced by biomechanical forces" due to either a direct or indirect blow, resulting in the impairment of neurologic function with clinical symptoms. ${ }^{5}$ The diagnosis of concussion is particularly challenging because acute symptoms, which are likely caused by functional disturbances rather than gross structural injury, are associated with normal standard structural neuroimaging (e.g., computed tomography scan). ${ }^{5}$ Symptoms of concussion may overlap with more severe pathologic traumatic brain injury or mimic other medical conditions, such as posttraumatic stress disorder, depression, and headache syndromes. ${ }^{6-10}$ The identification of concussion is critical to deliver appropriate care and support to patients and their families. ${ }^{11-13}$ Persistent symptoms following concussion can have a devastating impact. ${ }^{14,15}$ Consequences of concussion can include school absenteeism, depression, loss of social activities, and lower quality of life. ${ }^{16}$ Children and adolescents may miss weeks or even months of their school year, which can affect marks and jeopardize a promotion to the next grade level. ${ }^{17,18}$ Slowed cognition, decreased attention, and memory impairment may make schoolwork a challenge upon return and require educational interventions. $^{12,15,19,20}$ Given the serious short- and long-term effects following mild traumatic brain injury, the application of appropriate initial management practices is essential. ${ }^{21-26}$

Numerous guidelines for the management of concussion have been released from specialty groups (including sports medicine, neurology, pediatrics, and family medicine) and from large medical associations. There is a collective agreement across all guidelines that the initial management should include removal from play, physical and cognitive rest, and graduated return to normal activities following medical clearance. ${ }^{5,27-32}$ The 2012 Zurich Consensus statement (of the 4th
International Conference on Concussion in Sport) concluded that there should be no return to play on the day of injury for the child or adolescent athlete, and it recommended a stepwise progression to return to play. Cognitive rest was described as a need to modify school attendance and activities. ${ }^{5}$ This consensus statement noted that it is appropriate to have an extended time of asymptomatic rest in children and adolescents. Similarly, the American Academy of Neurology guidelines recommend a more conservative return to play in children and adolescents than in adults, but the guidelines do not specify cognitive rest. ${ }^{30}$

The incidence of pediatric concussion has increased by over $50 \%$ in the past decade, resulting in a proportional increase in pediatric emergency department (ED) visits. There are approximately 250,000 pediatric concussion ED visits annually in the United States. ${ }^{33}$ The objective of this study was to examine the ability of Canadian pediatric emergency physicians to diagnose a concussion and evaluate the scope of practice variation for recommendations of physical and cognitive rest. The authors hypothesized that wide practice variation exists among pediatric emergency medicine physicians in the application of physical and cognitive rest recommendations following an acute concussion.

\section{MATERIALS AND METHODS}

\section{Study design}

A survey of Canadian pediatric emergency physicians was carried out to assess the knowledge of pediatric concussion and initial management, specifically regarding the application of physical and cognitive rest.

\section{Instrument development}

A multidisciplinary team of physicians with expertise in concussion diagnosis and management, pediatric emergency medicine, neuropsychology, allied health, assessment tool design, and medical education research established the questionnaire's length, format, and content. Four domains were identified: 1) knowledge of concussion diagnosis; 2) initial management using return-to-play/school/work recommendations (including subgroups B1: same-day return-to-play, B2: graduated return-to-play, and B3: cognitive rest [e.g., school attendance, homework, reading, screen time]); 3) knowledge of existing concussion guidelines or 
recommendations; and 4) perceived barriers to guideline use.

The team developed three case vignettes featuring common pediatric minor head injury scenarios of varying complexity (Appendix 1). The first vignette described a preadolescent male who developed a headache, dizziness, visual disturbances, and mild cognitive changes after an indirect blow to the head caused by an accidental collision to his chest during a hockey game. His symptoms partially improved over the 20 minutes subsequent to the collision. The purpose of this vignette was to determine whether a concussion would be recognized without a direct blow to the head and to determine the knowledge of same-day return-toplay contraindications. The second vignette described an adolescent female who developed amnesia, dizziness, and nausea after a nonsports-related fall at school in which her head struck a wall in a stairwell. The purpose of this vignette was to determine recognition of a nonsports concussion and to ensure that recommendations were made for cessation of physical exertion (even noncontact such as running) until the child was asymptomatic. The final vignette described an adolescent male who had a football helmet-to-helmet collision resulting in a 1-minute loss of consciousness, amnesia, headache, vision changes, and nausea. This classic case was included to correspond with the more constrained pre-2002 definition of a concussion. ${ }^{4}$ All three vignettes exceeded the lowest threshold for the diagnosis of a concussion. ${ }^{4,5}$ To assess the respondents' knowledge of concussion diagnosis and management, four closedended questions were posed for each of the case vignettes. The first question inquired whether the child suffered a concussion (correct answer: yes). Subsequent questions queried whether same-day return to play was possible (correct answer: no) or whether athletic competitions were possible in the next 2 to 5 days (correct answer: no), and suggested cognitive rest options (choices included school absence; reduction of schoolwork; and avoidance of computer use, text messaging, playing video games, and watching the television). Five demographic questions were also included. Prior to distribution, the questionnaire was pilot tested for clarity and appropriateness by 10 emergency physicians and members of the Concussions Ontario: Diagnosis and Early Education working group (which included allied health and physician representatives) and revised as necessary. ${ }^{34}$ All reviewers were excluded from the participant population.

\section{OUTCOMES}

The primary outcome was the application of the Zurich Consensus definition to diagnose a concussion. Secondary outcomes included practice variation for physical rest recommendations (no same-day return-tosport and graduated return-to-play) and practice variation for cognitive rest.

\section{Participants}

The authors enrolled pediatric emergency medicine physicians who are members of the Pediatric Emergency Research Canada (PERC). PERC is a network of 15 pediatric or teaching hospitals across Canada with diverse geographic membership: 38\% from Ontario, 21\% from Alberta, 17\% from Quebec, 8\% from Nova Scotia, 5\% from British Columbia, 4\% from Manitoba, $3 \%$ from Saskatchewan, and 3\% from Newfoundland. After the exclusion of study investigators and pilot survey participants $(n=6)$, the questionnaire was distributed to the remaining 176 PERC members. Survey respondents who were not attending physicians or did not regularly diagnose or manage children and adolescents with concussions were excluded. ${ }^{34}$

\section{Ethical considerations}

Prior to survey distribution, the study was approved by the PERC Executive Committee and the Research Ethics Board of the Children's Hospital of Eastern Ontario. This project was funded by a grant from the Ontario Neurotrauma Foundation, Toronto, Ontario, Canada.

\section{Data collection procedure}

The questionnaire and accompanying information letter were distributed electronically from November 15, 2012, to January 15, 2013, using FluidSurveys (Fluidware, Ottawa, ON), a Canadian Web-based questionnaire system. ${ }^{35}$ Based on a modified version of Dillman's tailored design method, ${ }^{36}$ two reminder emails were sent in an attempt to maximize the response rate. All questionnaire responses were kept confidential, and only aggregated data were reported.

\section{Data analysis}

Analyses of the questionnaire data were performed using the statistical software tool, SPSS (Version 20). ${ }^{37}$ 
Standard descriptive statistics were calculated, with 95\% confidence intervals (CI) using the Wilson method when appropriate.

\section{RESULTS}

\section{Characteristics of respondents}

In total, 115 health care providers $(65 \%)$ responded to the questionnaire; 109 providers replied to questions for all three case vignettes; and 108 answered all questions for the three vignettes. Of those, 105 provided demographic information and identified their highest level of postgraduate training in pediatric emergency medicine $(n=76)$, pediatrics $(n=16)$, emergency medicine $(n=11)$, family medicine $(n=1)$, and sports medicine $(n=1)$. Three respondents declined to provide demographic information. The great majority of respondents (89\% [95\% CI: 0.81, 0.93]) reported having diagnosed greater than 20 concussions per year. Demographic

\begin{tabular}{|c|c|c|}
\hline Item & $N$ & $n(\%)$ \\
\hline Number of concussions diagnosed annually & 105 & \\
\hline$<5$ & & $1(1)$ \\
\hline $5-10$ & & $2(2)$ \\
\hline $11-15$ & & $2(2)$ \\
\hline $16-20$ & & $7(7)$ \\
\hline$>20$ & & $93(89)$ \\
\hline Number of years practicing independently & 105 & \\
\hline$<5$ & & $30(29)$ \\
\hline $5-10$ & & $26(25)$ \\
\hline $11-15$ & & $24(23)$ \\
\hline $16-20$ & & $11(11)$ \\
\hline$>20$ & & $14(13)$ \\
\hline Population of catchment area & 105 & \\
\hline$<50000$ & & $1(1)$ \\
\hline $50000-100000$ & & $0(0)$ \\
\hline $100001-500000$ & & $13(12)$ \\
\hline $500001-1000000$ & & 19 (18) \\
\hline$>1000000$ & & 72 (69) \\
\hline Primary work setting & 105 & \\
\hline Solo practice & & $0(0)$ \\
\hline Group practice & & $0(0)$ \\
\hline Academic practice (educational institution) & & $33(31)$ \\
\hline Private practice & & $0(0)$ \\
\hline Hospital setting & & $6(6)$ \\
\hline Walk-in or Acute Care clinic & & $0(0)$ \\
\hline Emergency Department & & $66(63)$ \\
\hline Other & & $0(0)$ \\
\hline
\end{tabular}

characteristics of the study participants are provided in Table 1.

Knowledge of concussion diagnosis and understanding of initial management using return-to-play guidelines

The great majority of respondents (98 out of 109; $90 \%$ [95\% CI: $0.83,0.94]$ ) were able to properly diagnose a concussion correctly in all three clinical vignettes. Similarly, 102 of 108 (94\% [95\% CI: 0.87, 0.97]) of respondents correctly applied the recommended guidelines to disallow return to play on the same day of injury (domain B1). ${ }^{5,30}$ Respondents demonstrated a larger practice variation in the application of graduated returnto-play recommendations in a conservative stepwise progression, defined as 24 hours between successive steps. Correct application would result in an asymptomatic patient requiring 1 week to proceed through the six steps ${ }^{5}$ (domain B2). Only 69 of 108 respondents (64\% [95\% CI: $0.54,0.72])$ applied a conservative graduated return-toplay recommendation in all three vignettes when queried about the possibility of return to full-contact sport competition at day four. For example, for vignette one, $33 \%$ of respondents would have possibly allowed a child to return to a hockey tournament 4 days after the initial concussion. In vignette two, $12 \%$ would have possibly allowed the adolescent to participate in a $5-\mathrm{km}$ running race in 2 days. For vignette three, only 7\% would have allowed participation in a hockey game in 4 days. Permission (or possible permission) to participate in these activities was deemed to be an incorrect application of conservative stepwise progression.

\section{Practice variation of recommendations for cognitive rest}

Respondents showed wide practice variation in recommendations for cognitive rest (domain B3, Table 2). Of note, $35 \%$ of respondents (95\% CI: $0.27,0.45$ ) did not recommend a reduction of screen time (aggregate of computer, text messaging, video game, and television reductions). In total, only 40 of 108 (37\%, 95\% CI: $0.29,0.46)$ recommended thorough cognitive rest, defined as a reduction of school, schoolwork, and all forms of screen time (Appendix 2 [Supplementary table of the remaining recommendations]).

\section{Use of existing concussion protocols}

Only 22\% (95\% CI: $0.15,0.30)$ of respondents reported having used common concussion scoring scales frequently 
Table 2. Participant recommendations for cognitive rest

\begin{tabular}{|c|c|c|c|}
\hline Item & $N$ & $n$ & $\begin{array}{l}\text { Proportion, \% } \\
\qquad(95 \% \mathrm{Cl})\end{array}$ \\
\hline Cognitive rest recommendations & 108 & & \\
\hline Stay home from school & & 65 & $60 \%(0.51,0.69)$ \\
\hline Avoid computer screen & & 85 & $79 \%(0.70,0.85)$ \\
\hline Avoid text messaging & & 75 & $69 \%(0.60,0.77)$ \\
\hline Avoid video games & & 88 & $81 \%(0.73,0.88)$ \\
\hline Limit television & & 86 & $80 \%(0.71,0.86)$ \\
\hline Decrease schoolwork & & 76 & $70 \%(0.61,0.78)$ \\
\hline Rest in a quiet, dark environment & & 58 & $54 \%(0.44,0.63)$ \\
\hline $\begin{array}{l}\text { Perfect screen-time advice (aggregate of computer, } \\
\text { text message, video game, and television reduction) }\end{array}$ & & 70 & $65 \%(0.55,0.73)$ \\
\hline
\end{tabular}

\begin{tabular}{|lrr}
\hline Table 3. Concussion scoring scales used "frequently" or "Always" & $n$ & Proportion, \% (95\% Cl) \\
\hline Concussion scoring scale & $N$ & $n$ \\
$\quad$ Standardized Assessment of Concussion & 106 & $2 \%(0.01,0.07)$ \\
Sport Concussion Assessment Tool & 2 & $17 \%(0.11,0.25)$ \\
Post-concussion Symptom Scale & 18 & $1 \%(0.002,0.05)$ \\
Graded Symptom Checklist/Scale & 3 & $3 \%(0.01,0.08)$ \\
McGill Abbreviated Concussion Evaluation & 2 & $2 \%(0.01,0.07)$ \\
Head Injury Scale & 0 & $0 \%(0.00,0.03)$ \\
Post-concussion Symptom Inventory & 1 & $1 \%(0.002,0.05)$ \\
Rivermead Post-concussion Symptom Questionnaire & 0 & $0 \%(0.00,0.03)$
\end{tabular}

\begin{tabular}{|c|c|c|c|}
\hline Item & $N$ & $n$ & Proportion, \% (95\% Cl) \\
\hline Guideline/Policy & 106 & & \\
\hline 1. Hospital/Practice policy & & 49 & $46 \%(0.37,0.56)$ \\
\hline 2. American Academy of Neurology & & 28 & $26 \%(0.19,0.36)$ \\
\hline 3. Cantu Guidelines & & 4 & $4 \%(0.01,0.09)$ \\
\hline 4. Centre for Disease Control & & 6 & $6 \%(0.03,0.12)$ \\
\hline 5. Colorado Medical Society & & 1 & $1 \%(0.00,0.05)$ \\
\hline 6. $3^{\text {rd }}$ International Conference on Concussion in Sport (Zurich) & & 40 & $38 \%(0.29,0.47)$ \\
\hline 7. $2^{\text {nd }}$ International Conference on Concussion in Sport (Prague) & & 9 & $8 \%(0.05,0.15)$ \\
\hline 8. $1^{\text {st }}$ International Symposium on Concussion in Sport (Vienna) & & 2 & $2 \%(0.00,0.07)$ \\
\hline 9. Roberts & & 0 & $0 \%(0.00,0.03)$ \\
\hline 10. ThinkFirst Canada & & 56 & $53 \%(0.43,0.62)$ \\
\hline 11. Ontario Neurotrauma Foundation mTBI guidelines & & 3 & $3 \%(0.01,0.08)$ \\
\hline 12. Canadian Academy of Sports Medicine & & 31 & $29 \%(0.21,0.39)$ \\
\hline 13. Other & & 18 & $17 \%(0.11,0.25)$ \\
\hline
\end{tabular}

or always (Table 3); 70\% (95\% CI: 0.61, 0.78) of respondents reported having used balance-testing tools frequently or always. In addition, $100 \%$ (95\% CI: $0.97,1.00)$ of respondents reported that they do not use computerized testing tools. Most (80\% [95\% CI: 0.72, 0.87]) reported that they use published guidelines frequently or always to guide clinical decisions regarding concussion, with only one person ( $1 \%$ [95\% CI: 0.00, 0.05]) reporting having never used guidelines. Table 4 summarizes the reported use of commonly known concussion guidelines/policies. 


\section{Conclusions}

Only $18 \%$ (95\% CI: $0.12,0.26)$ of respondents reported that they frequently or always refer patients to concussion specialists. Respondents who refer to concussion specialists most often refer to sports medicine specialists (67\% [95\% CI: 0.57, 0.75]) and neurologists (50\% [95\% CI: $0.41,0.60])$. The most common barrier reported by those who never refer to concussion specialists (3\% [95\% CI: $0.01,0.08]$ ) is the lack of concussion specialists in their region (67\% [95\% CI: 0.21, 0.94]).

\section{DISCUSSION}

\section{Summary}

This is the first broad Canadian analysis of pediatric emergency medicine physicians' knowledge of concussion diagnosis and initial management practice patterns. The results in this study indicate that knowledge of concussion diagnosis is adequate for pediatric emergency providers in Canada. However, a wide-practice variation for the appropriate use of physical rest (return-to-play) recommendations and cognitive rest advice was found.

Although several previous studies demonstrate that knowledge gaps exist in the diagnosis and management of concussion by athletes, coaches, and trainers, ${ }^{38-40}$ few have examined practicing physicians' knowledge of concussion diagnosis and early management. The findings in this study are consistent with other literature, suggesting that concussion knowledge and management deficiencies are pervasive. Recently, Zonfrillo et al. examined the self-reported knowledge and attitudes of 145 pediatricians and pediatric emergency physicians affiliated with a single institution at an urban, academic, tertiary-care centre in the United States, and reported that inadequate knowledge existed of pediatric concussion diagnosis and initial management. ${ }^{41}$ Boggild and Tator reported that gaps in general knowledge of concussion (e.g., concussion definition, signs/symptoms, initial management, and sequelae) existed in a survey of graduating medical students and postgraduate trainees in neurology and neurosurgery. ${ }^{42}$ Lebrun et al. found that family physicians in Alberta and North Dakota rarely recommended cognitive rest $(47.5 \%$ and $28 \%$, respectively), despite existing guidelines. ${ }^{43}$ Bazarian et al. examined pediatric, family, and emergency providers in Rochester, New York, on their knowledge of concussion grading and similarly found that the correct application of return-to-play guidelines was below 57\%. That study examined knowledge of the pre-2002 definition of concussion. ${ }^{44}$ Giebel et al. similarly noted a low rate of guideline use $(23 \%)$ in a survey of emergency providers in Kalamazoo, Michigan. ${ }^{45}$

Given strong recommendations by the Zurich Consensus and American Academy of Neurology for a more conservative application of return-to-sport guidelines in children and adolescents, ${ }^{5,30}$ it seems clear that further knowledge translation is required to improve clinician application of guidelines. The development and implementation of easy-to-use tools and management pathways have been demonstrated to be effective in aligning clinical practice with the best available evidence. Integrated knowledge translation with key end-users and stakeholders is essential to permit broad implementation of future guidelines for concussion.

This study reveals wide practice variation regarding cognitive rest advice. There is little evidence beyond expert opinion for the ideal cognitive and physical recommendations, and the observed wide practice variation may reflect the lack of high level (level A or B) evidence in this area. ${ }^{46}$ A comparative trial is needed for both return-to-activity and return-to-school guidelines to determine the ideal components to improve outcomes after concussion.

This study employed a broad distribution in an effort to obtain a baseline understanding of how concussion is currently being diagnosed and managed. The majority of respondents indicated that they diagnose more than 20 concussions per year; therefore, our results reflect the practice for in excess of 2000 acute pediatric concussions across Canada annually. This study is the first examining practicing pediatric emergency providers' practice variation of cognitive rest. Zonfrillo et al. examined the barriers to providing education to families following a pediatric concussion across pediatric providers at the Children's Hospital of Philadelphia, but they did not describe the practice variation of cognitive rest instructions provided. ${ }^{41}$ The development of our questionnaire was systematic, and it strengthened by involving a group of experts from various fields.

\section{Limitations}

The nature of this study involved the potential for selection bias, specifically that those who chose to respond may have been more likely to have a greater 
Pediatric emergency physician concussion knowledge

concussion knowledge or familiarity. It has been found that in surveys of the health care professionals, response rates are often influenced by respondents' characteristics and high workload demands. ${ }^{47}$ Other limitations are similarly inherent to the use of surveys examining practice variation. For example, respondents may not practice in the manner that they indicate in the survey. Recall that bias may also have been a factor in this study's findings (e.g., physicians may under- or overreport the number of concussion patients treated per year). The potential for variation in the interpretation of graduated scale questions also exists (e.g., different respondents may have different interpretations of the words sometimes or frequently). A prospective examination of discharge instructions by providers would provide a more accurate representation of practice variation. The lack of a single gold standard guideline for pediatric concussion is also a limitation, which is further limited by the large proportion of level C (expert opinion) evidence related to return-to-play and cognitive rest recommendations. There is a need for a large comparative trial examining the benefit of applying gradations of rest. The authors recognize that it is likely that the majority of children in Canada with a concussion are treated by general practitioners and at non-tertiary EDs. A recent study estimated the incidence of concussion to be approximately 6 of $1000 .^{48}$ The authors' team carried out a similar study of family doctors, general emergency physicians, nurse practitioners, and general pediatricians practicing in Ontario and found that similar gaps in knowledge existed across all provider types. ${ }^{49}$

\section{Conclusion}

Gaps in knowledge and broad practice variation in recommending graduated return to play and cognitive rest following concussion exist among Canadian pediatric emergency physicians. The development and implementation of easy-to-use pediatric-specific concussion guidelines and management pathways have the potential to standardize clinical practice based on bestavailable evidence. Integrated knowledge translation with key end-users and stakeholders is essential to encourage the broader implementation of future pediatric concussion guidelines. Future research should address how best to overcome barriers to knowledge uptake and protocol application for the diagnosis and management of pediatric concussion.
Competing interests: None declared.

\section{SUPPLEMENTARY MATERIAL}

To view supplementary material for this article, please visit http://dx.doi.org/10.1017/cem.2014.38

\section{REFERENCES}

1. Naunheim RS, Matero D, Fucetola R. Assessment of patients with mild concussion in the emergency department. 7 Head Trauma Rebabil 2008;23(2):116-22.

2. Guskiewicz KM, Register-Mihalik J, McCrory P, et al. Evidence-based approach to revising the SCAT2: introducing the SCAT3. Br 7 Sports Med 2013;47(5):289-93.

3. McCrea M, Iverson GL, Echemendia RJ, et al. Day of injury assessment of sport-related concussion. $\mathrm{Br} 7$ Sports Med 2013;47(5):272-84.

4. McCrory P, Meeuwisse WH, Echemendia RJ, et al. What is the lowest threshold to make a diagnosis of concussion? $\operatorname{Br} 7$ Sports Med 2013;47(5):268-71.

5. McCrory P, Meeuwisse WH, Aubry M, et al. Consensus statement on concussion in sport: the 4th International Conference on Concussion in Sport held in Zurich, November 2012. Br 7 Sports Med 2013;47:250-8.

6. Ayr LK, Yeates KO, Taylor HG, et al. Dimensions of postconcussive symptoms in children with mild traumatic brain injuries. 7 Int Neuropsychol Soc 2009;15(01):19.

7. Ponsford J, Willmott C, Rothwell A, et al. Cognitive and behavioral outcome following mild traumatic brain injury in children. 7 Head Trauma Rehabil 1999;14(4):360-72.

8. Yeates KO, Luria J, Bartkowski H, et al. Postconcussive symptoms in children with mild closed head injuries. 7 Head Trauma Rebabil 1999;14(4):337-50.

9. Gioia GA, Collins M, Isquith PK. Improving identification and diagnosis of mild traumatic brain injury with evidence: psychometric support for the acute concussion evaluation. 7 Head Trauma Rebabil 2008;23(4):230-42.

10. Kashluba S, Paniak C, Casey JE. Persistent symptoms associated with factors identified by the WHO Task Force on mild traumatic brain injury. Clin Neuropsychol 2008;22(2):195-208.

11. Taylor HG, Dietrich A, Nuss K, et al. Post-concussive symptoms in children with mild traumatic brain injury. Neuropsychology 2010;24(2):148-59.

12. Babikian T, Satz P, Zaucha K, et al. The UCLA longitudinal study of neurocognitive outcomes following mild pediatric traumatic brain injury. I Int Neuropsychol Soc 2011;17(05):886-95.

13. Ganesalingam K, Yeates KO, Ginn MS, et al. Family burden and parental distress following mild traumatic brain injury in children and its relationship to post-concussive symptoms. 7 Pediatr Psychol 2007;33(6):621-9.

14. Carroll L, Rosner D. The concussion crisis: anatomy of a silent epidemic. New York Simon \& Schuster; 2011.

15. Yeates KO, Kaizar E, Rusin J, et al. Reliable change in postconcussive symptoms and its functional consequences among children with mild traumatic brain injury. Arch Pediatr Adolesc Med 2012;166(7):615-22. 
16. Scorza KA, Raleigh MF, O'Connor FG. Current concepts in concussion: evaluation and management. Am Fam Physician 2012;85(2):123-32.

17. Meehan WP, Bachur RG. Sport-related concussion. Pediatrics 2009;123(1):114-23.

18. Meehan WP, Mannix R. Pediatric concussions in United States Emergency Departments in the years 2002 to 2006. 7 Pediatr 2010;157(6):889-93

19. Forsyth R, Kirkham F. Predicting outcome after childhood brain injury. CMA7 2012;184(11):1257-64.

20. Korinthenberg R, Schreck J, Weser J, et al. Post-traumatic syndrome after minor head injury cannot be predicted by neurological investigations. Brain Dev 2004;26(2):113-7.

21. Marchie A, Cusimano MD. Bodychecking and concussions in ice hockey: should our youth pay the price? CMAJ 2003;169(2):124-8.

22. Guskiewicz K, Marshall S, Bailes J, et al. Association between recurrent concussion and late-life cognitive impairment in retired professional football players. Neurosurgery 2005;57(4):719-26.

23. Gaetz M, Goodman D, Weinberg H. Electrophysiological evidence for the cumulative effects of concussion. Brain Injury 2000;14:1077-88.

24. Gronwall D, Wrightson P. Cumulative effects of concussion. Lancet 1975;2:995-7.

25. Matser EJT, Kessels AG, Lezak MD, et al. Cumulative problems with memory and planning in amateur soccer. 7AMA 1999;282(10):971-3.

26. Swaine BR, Tremblay C, Platt RW, et al. Previous head injury is risk factor for subsequent head injury in children: a longitudinal cohort study. Pediatrics 2007;119:749-58.

27. Centers for Disease Control (CDC) Atlanta. Heads-up: a toolkit for physicians. Available at: http://www.cdc.gov/ concussion/headsup/pdf/Facts_for_Physicians_booklet-a.pdf (accessed October 30, 2013).

28. Purcell L. Canadian Pediatric Society, Healthy Active Living and Sports Medicine Committee. Evaluation and management of children and adolescents with sports-related concussion. 7 Paediatr Child Health 2012;17(1):31. Available at: http://www.cps.ca/documents/position/concussion-evaluationmanagement] (accessed October 30, 2013).

29. Halstead ME, Walter KD. American Academy of Pediatrics, Council on Sports Medicine and Fitness. Sport-related concussion in children and adolescents. Pediatrics 2010; 126(3):597-615.

30. Giza CG, Kutcher JS, Ashwal S, et al. Summary of evidencebased guideline update: evaluation and management of concussion in sports; Report of the Guideline Development Subcommittee of the American Academy of Neurology. Neurology 2013;80(24):2250-7.

31. Canadian Medical Association (CMA). 2011. Head injury and sport. Available at: http://policybase.cma.ca/dbtw-wpd/ Policypdf/PD11-10.pdf (accessed October 30, 2012).
32. Marshall S, Bayley M, McCullagh, et al. Clinical practice guidelines for mild traumatic brain injury and persistent symptoms. Can Fam Physician 2012;58(3):257-67.

33. Gilchrist J, Thomas $\mathrm{KE}, \mathrm{Xu} \mathrm{L}$, et al. Nonfatal traumatic brain injuries related to sports and recreation activities among persons $\leq 19$ years-United States, 2001-2009. MMWR Morb Mortal Wkly Rep 2011;60(39):1337-42.

34. Burns KEA, Duffett M, Kho ME, et al. A guide for the design and conduct of self-administered surveys of clinicians. CMA7 2008;179(3):245-52.

35. Inc Ci. FluidSurveys. 4.0 ed. Ottawa2012. p. Online Survey Software.

36. Dillman D. Mail and Internet surveys: the tailored design method. New York: John Wiley \& Sons; 2000.

37. IBM. SPSS Software. 20 ed. Armonk2012. p. Predictive analytics software and solutions.

38. Provvidenza C, Engebretsen L, Tator C, et al. From consensus to action: knowledge transfer, education and influencing policy on sports concussion. Br 7 Sports Med 2013;47(5):332-8.

39. Valovich McLeod TC, Schwartz C, Bay RC. Sport-related concussion misunderstandings among youth coaches. Clin 7 Sport Med 2007;17:140-2.

40. Sye G, Sullivan J, McCrory P. High school rugby players' understanding of concussion and return to play guidelines. Br 7 Sports Med 2006;40:1003-5.

41. Zonfrillo MR, Master CL, Grady MF, et al. Pediatric providers' self-reported knowledge, practices, and attitudes about concussion. Pediatrics 2012;130(6):1120-5.

42. Boggild MM, Tator CH. Concussion knowledge among medical students and neurology/neurosurgery residents. Can 7 Neurol Sci 2012;39(3):361-8.

43. Lebrun CM, Mrazik M, Prasad AS, et al. Br 7 Sports Med 2013;47:54-9.

44. Bazarian JJ, Veenema T, Brayer AF, et al. Knowledge of concussion guidelines among practitioners caring for children. Clin Pediatr (Phila) 2001;40:207-12.

45. Giebel S, Kothari R, Koestner A, et al. Factors influencing emergency medicine physicians' management of sportsrelated concussions: a community-wide study. 7 Emerg Med 2011;41(6):649-54.

46. DeMatteo CA, Hanna SE, Mahoney WJ, et al. "My child doesn't have a brain injury, he only has a concussion. Pediatrics 2010;125(2):327-34.

47. Hill CA, Fahrney K, Wheeless SC, et al. Survey response inducements for registered nurses. West 7 Nurs Res 2006; 28(3):322-34.

48. Ryu WH, Feinstein A, Colantonio A, et al. Early identification and incidence of mild TBI in Ontario. Can 7 Neurol Sci 2009;36:429-35.

49. Zemek R, Eady K, Moreau K, et al. Knowledge of Paediatric Concussion in Front-Line Primary Care Providers. Paediatr Child Health 2014;19(9):475-80. 